\title{
The Pyramid of Injury: Estimation of the Scale of Adolescent Injuries According to Severity
}

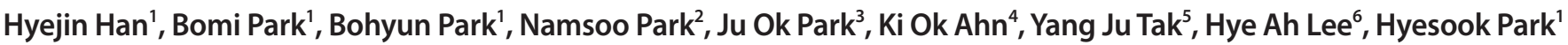 \\ ${ }^{1}$ Department of Preventive Medicine, Ewha Womans University School of Medicine, Seoul, Korea; ${ }^{2}$ Department of Health Management, Hyupsung \\ University, Hwaseong, Korea; ${ }^{3}$ Department of Emergency Medicine, Hallym University College of Medicine and Dongtan Sacred Heart Hospital, \\ Hwaseong, Korea; ${ }^{4}$ Department of Emergency Medicine, Myoungji Hospital, Goyang, Korea; ${ }^{5}$ Department of Paramedic Science, Korea National \\ University of Transportation, Chungju, Korea; ${ }^{6}$ Clinical Trial Center, Ewha Womans University Mokdong Hospital, Seoul, Korea
}

Objectives: Due to their developmental characteristics, adolescents have a higher probability than other age groups of experiencing injuries caused by accidents, violence, and intentional self-harm. The severity and characteristics of injuries vary by the intentionality and mechanism of injury; therefore, there is a need for a national-level estimate of the scale and the severity of injuries in adolescents that takes these factors into account.

Methods: By using data from the Emergency Department-based Injury In-depth Surveillance Data, National Emergency Department Information System, the Korean National Hospital Discharge In-depth Injury Survey, and cause of death statistics, we calculated the emergency department (ED) visit rate, hospitalization rate, and death rate of injuries per 100000 adolescents for each injury mechanism. The calculated rates were used to generate the injury pyramid ratio (ratio of death rate to hospitalization rate to ED visit rate) to visualize the scale and the severity of the injury.

Results: The mortality rate in adolescents due to injury was 10/100 000; the corresponding rates for hospitalization and ED visits were 1623 and 4923, respectively, resulting in an injury pyramid ratio with the general pyramid form, with a 1:162:492 ratio of deaths to hospitalizations to ED visits. The mortality rate due to suicide/intentional self-harm was 5/100 000, while 35 were hospitalized for this reason and 74 made ED visits. The pyramid ratio of 1:7:15 for intentional self-harm/suicide showed a steep pyramidal form, indicating considerable lethality. The mortality rate due to motor vehicle collisions (MVCs) was 3/100 000; 586 were hospitalized for this reason, while 1023 made ED visits. The pyramid ratio of 1:195:341 for MVCs showed a gradual pyramid form, indicating that the lethality was low and the scale of injury was high.

Conclusions: The main categories of injuries in adolescents were visualized in pyramid form, contributing to an understanding of the scale of each injury by mechanism in terms of levels of death, hospitalization, and ED visits. These findings will be helpful for understanding how to prioritize injuries in adolescents.

Key words: Adolescent, Injury, Severity, South Korea

Received: January 31, 2018 Accepted: May 23, 2018

Corresponding author: Hyesook Park, MD, PhD

Department of Preventive Medicine, Ewha Womans University School of Medicine, 1071 Anyangcheon-ro, Yangcheon-gu, Seoul 07985,

Korea

E-mail: hpark@ewha.ac.kr

This is an Open Access article distributed under the terms of the Creative Commons Attribution Non-Commercial License (http://creativecommons.org/licenses/by$\mathrm{nc} / 4.0 /$ ) which permits unrestricted non-commercial use, distribution, and reproduction in any medium, provided the original work is properly cited.

\section{INTRODUCTION}

Injury is a major global health concern. Despite differences across countries in the most widespread mechanisms of injury based on each country's health status and social environment, injury in general is regarded as a high-priority issue in most countries [1-4]. In a study of the burden of disease in South Korea (hereafter Korea), injuries such as falls, motor vehicle collisions (MVCs), and suicides were included in the top 10 
causes of disability-adjusted life years [5].

Adolescents are at higher risks of injuries occurring from accidents, violence, and intentional self-harm/suicide [6], and their burden of disease due to injuries is higher than that of other age groups [7]. According to the 2016 Statistics on the Youth, the top first and second causes of death in adolescents between 2008 and 2014 were intentional self-harm/suicide and MVCs [8].

The concept of the injury pyramid was first used in Heinrich et al. [9] study on industrial accident prevention published in the 1930s. Since then, the concept has been applied and supplemented, and is now used as a model for studies of injuries $[10,11]$. The injury pyramid is a visual representation of the burden of disease caused by injuries. The bottom section of the pyramid shows the rate of emergency department (ED) visits, the middle section represents the rate of hospitalization, and the top section represents deaths caused by injury. The visualization of the pyramid ratio of deaths to hospitalizations to ED visits can then be used to depict the scale and severity of injuries; therefore, the pyramid ratio can be used as an indicator that enables an intuitive understanding of the scope of each type of injury [12].

This study aims to visualize injuries in adolescents, a group vulnerable to injury, using the injury pyramid ratio based on data sources collected from hospitals in Korea, so that the scale and severity of injuries in this population can be easily understood and interpreted.

\section{METHODS}

The study subjects were injured adolescents (ages 13-18) who were selected by using the 2015 data on cause of death $(n=360)$, the 2013-2015 Korean National Hospital Discharge In-depth Injury Survey (KNHDS) ( $n=61$ 723), the Emergency Department-based Injury In-depth Surveillance Data (EDIS) $(n=16352)$, and National Emergency Department Information System (NEDIS) ( $n=157$ 933).

Data from Statistics Korea were used to collect data on injuries that caused death in adolescents. Statistics Korea provides a national index of death rates per 100000 people based on death reports that are released annually. Injuries are defined using the disease classification codes V01-Y89 (contraction of disease and unnatural death) under the Korean Standard Classification of Diseases sixth revision (KCD-6). Injuries are more specifically categorized as unintentional injury (V01-X59) and intentional injury (self-harm/suicide, X60-84; violence, X85Y09) by the intentionality of injuries and by the mechanism of injuries, such as MVCs (V01-99), poisoning (X40-49), falls (W00-19), struck by/against (W20-24, W27-31, W35-40, W45, W49-52, W54-64), fire/burns (X00-19), and drowning (W6574). The death rate caused by each injury per 100000 people was calculated by dividing the number of deaths from that injury by the mid-year population of registered residents [13].

Data from the KNHDS conducted by the Korean Centers for Disease Control and Prevention (KCDC) were used to investigate hospitalizations due to injury. Nine percent of the annually discharged patients from 170 acute-care hospitals with an electronic medical record system, which were sampled by geographic location and number of beds out of general hospitals with 100 or more beds, were randomly sampled and their medical records were reviewed. Injuries were defined using the principal or secondary diagnosis code of S00-T98 in the KCD-6 and injuries were categorized by the mechanism, as we did for cause of death. Cut/pierce injuries (W20-27, W29, W45, W49, W53, W54-64) were additionally defined. In order to obtain a sufficient number of participants, data from the recent 3 years (surveyed years from 2011 to 2013) were combined, and weighting for the complex sample survey was applied for each year. The weighted frequency of discharged patients for each injury mechanism was divided by the standard population (2011-2013 average mid-year population of registered residents) to calculate the hospitalization rate per 100000 population [14].

The NEDIS and the EDIS were used to investigate ED visits due to injury. The NEDIS is updated in real-time by the National Emergency Medical Center across Korea. Developed in 2004, the database information is drawn from EDs throughout Korea. The EDIS is conducted by the KCDC and investigates the mechanism and cause of injury in injured patients who were hospitalized after their initial ED visits in the participating 23 hospitals. For this study, injuries were categorized according to intentionality as unintentional (C1) or intentional $(C 2,3)$ according to the 10th revision of the International Statistical Classification of Diseases and Related Health Problems code. Injury mechanisms were defined as MVCs (C11), falls, (C12), struck by/against (C13), machines (C3), drowning (C5), and poisoning (C6.1), and the scale of injuries resulting in ED visits was calculated [15].

The rates of ED visits due to injury by mechanism were calculated using the NEDIS. To calculate the rates of ED visits due to sub-divided injury mechanisms, the EDIS was used. Howev- 
er, since the hospitals participating in the EDIS are selected by a simple random sampling without taking into account regional or hospital-specific characteristics, an extrapolation factor (EF) was applied to generate a nationally representative estimate. The EF was calculated by dividing the number of injured patients on a national level provided by the NEDIS by the number of injured patients provided by the EDIS [16]. Finally, the rate of ED visits by injury mechanism per 100000 people was calculated using the mid-year population of registered residents of that year.

The injury pyramid ratio was calculated by comparing the death rates, the hospitalization rates, and the ED visit rates, with death rates as a reference value. For direct comparison between rates from different data sources, the calculated number of patients hospitalized was weighted by 1.1 and the number of patients who visited the ED was weighted by 1.9. The representativeness of this method was confirmed in a previous study [17].

All data analysis was conducted using SAS version 9.4 (SAS Institute Inc., Cary, NC, USA).

\section{RESULTS}

In adolescents, the death rate, the hospitalization rate, and the ED visit rate due to injuries were 10.00 deaths, 1623 hospitalizations, and $4923 \mathrm{ED}$ visits (per 100000 people of all ages), respectively. The injury pyramid ratio for all injuries was 1:162:492 (Table 1).

Comparisons of the scale of death, hospitalizations, and ED visits based on the intentionality of injury showed that the rates of death from unintentional and intentional injuries were similar (4 vs. 5/100 000), while the hospitalization rate (1486 vs. $145 / 100000$ ) and ED visitation rate (4614 vs. $301 / 100000$ ) were significantly higher for unintentional injuries. The injury pyramid ratio was 1:372:1154, while the corresponding ratio for intentional injuries was 1:29:60, indicating a significantly higher lethality for intentional injuries.

Comparison of the scale and severity of specific injury mechanisms showed that MVCs had the greatest number of deaths (127/100 000), followed by falls (111/100 000). MVCs had the highest rate of hospitalization (586/100 000), followed by falls

Table 1. Injuries ${ }^{1}$ by various mechanisms: ED visits, hospitalization, and deaths in participants aged 13-18 years in Korea

\begin{tabular}{|c|c|c|c|c|c|c|c|}
\hline \multirow{2}{*}{$\begin{array}{l}\text { Injury } \\
\text { mechanism }\end{array}$} & \multicolumn{2}{|c|}{ ED visits } & \multicolumn{2}{|c|}{ Hospitalizations $^{2}$} & \multicolumn{2}{|c|}{ Deaths } & \multirow{2}{*}{$\begin{array}{c}\text { Pyramid ratio } \\
\text { (deaths:hospitalizations:ED visits) }\end{array}$} \\
\hline & Weighted $\mathbf{n}$ & Rate $^{3}$ & Weighted n & Rate $^{3}$ & Weighted n & Rate $^{3}$ & \\
\hline Total & 16305 & 4923 & 58567 & 1623 & 353 & 10.00 & 1:162:492 \\
\hline Unintentional injuries & 14595 & 4614 & 53621 & 1486 & 161 & 4.00 & $1: 372: 1154$ \\
\hline Intentional injuries & 1710 & 301 & 4946 & 145 & 192 & 5.00 & 1:29:60 \\
\hline Self-harm/suicide & 405 & 74 & 1260 & 35 & 178 & 5.00 & $1: 7: 15$ \\
\hline Violence & 1305 & 234 & 3686 & 102 & 14 & 0.39 & $1: 729: 1671$ \\
\hline \multicolumn{8}{|l|}{ Mechanism } \\
\hline MVCs & 3545 & 1023 & 21143 & 586 & 127 & 3.00 & 1:195:341 \\
\hline Bicycle $^{4}$ & 1490 & 431 & 3637 & 101 & 8 & 0.22 & 1:101:431 \\
\hline Motorcycle 4 & 871 & 252 & 7564 & 210 & 66 & 1.80 & 1:35:42 \\
\hline Falls & 3427 & 988 & 13771 & 382 & 111 & 3.00 & 1:127:329 \\
\hline Struck by/against & 5359 & 1301 & 9719 & 269 & 9 & $0.24^{5}$ & 1:1121:5421 \\
\hline Cut/pierce & 1411 & 656 & 2249 & 62 & - & - & - \\
\hline Machines & 14 & 12 & - & - & - & - & - \\
\hline Fire/burns & 280 & 136 & 1005 & 28 & 2 & $0.05^{5}$ & 1:560:2720 \\
\hline Drowning & 6 & 4 & 19 & 1 & 28 & 0.80 & $1: 1: 5$ \\
\hline Poisoning & 352 & 75 & 1433 & 40 & 13 & $0.35^{5}$ & $1: 114: 214$ \\
\hline
\end{tabular}

Data from: ED visits, Emergency Department Based Injury In-depth Surveillance, 2014 and National Emergency Department Information System, 2014 [15]; Hospitalization, Korean National Hospital Discharge In-depth Injury Survey, 2013-2015 [14]; Deaths, cause of death statistics, 2015 [13].

ED, emergency department; MVCs, motor vehicle collisions.

${ }^{1}$ The total injuries resulting in ED visits, hospitalizations, and deaths only included unintentional and intentional injuries, excluding unconfirmed and unknown injuries.

${ }^{2}$ The mechanism associated with hospitalization was analyzed based on a classification as unintentional injuries (except suicide and violence).

${ }^{3}$ Per 100000 people.

${ }^{4}$ The extrapolation method was only applied to ED visits.

${ }^{5}$ Rates based on fewer than 20 deaths may be unreliable. 


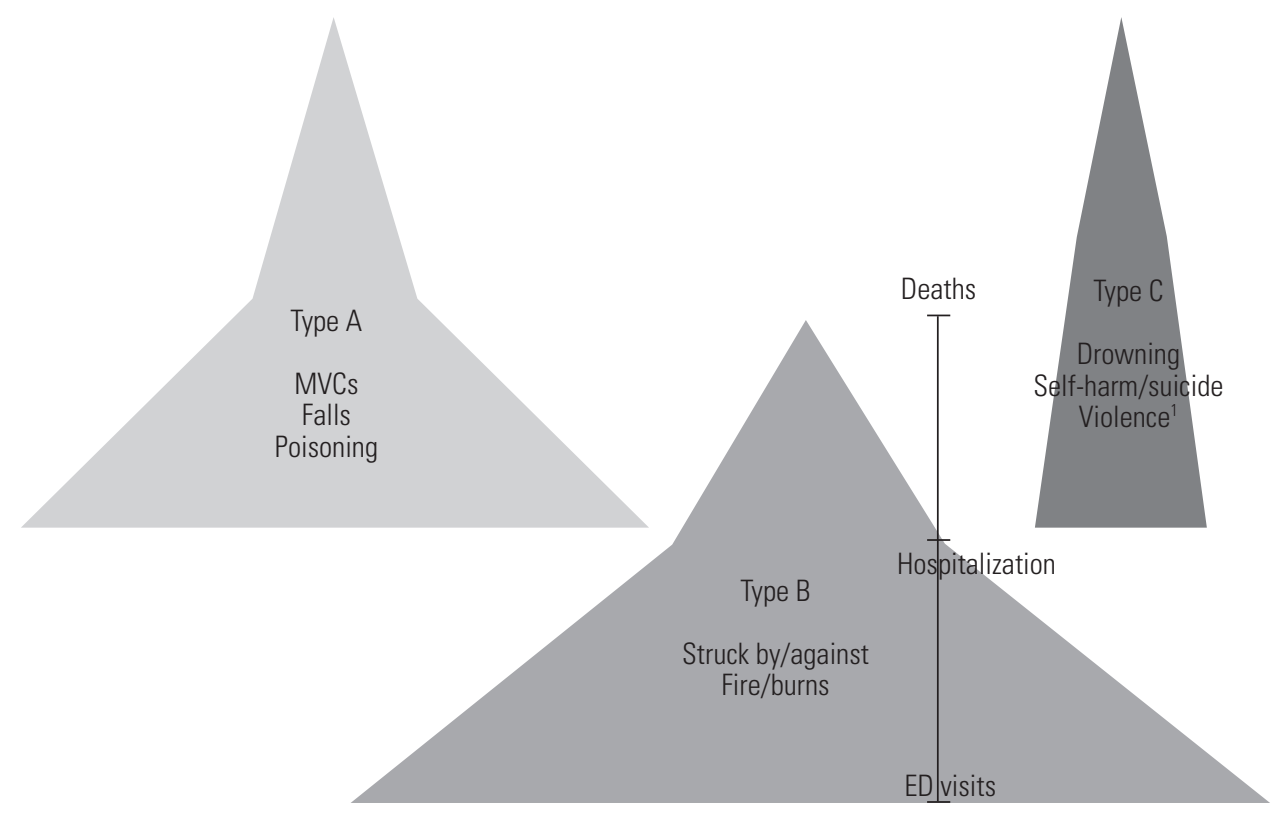

Figure 1. The injury pyramids 3 types (age 13-18, adolescent). MVCs, motor vehicle collisions; ED, emergency department. ${ }^{1} I n-$ tentional Injury.

(382/100 000), and struck by/against (269/100 000). Injuries due to the struck by/against mechanism had the highest rate of ED visits (1301/100 000), followed by MVCs (1023/100 000) and falls $(988 / 100000)$.

The pyramids for the various injury mechanisms in adolescents can be divided into 3 major types (Figure 1). While injuries by the struck by/against mechanism had the highest rate of ED visits, the hospitalization rate of this mechanism ranked third and its death rate was extremely low, with an injury pyramid ratio of 1:1121:5421. This indicates that the struck by/ against injury mechanism had a large scale of occurrence and showed great severity, but its lethality was very low. When shown in a pyramid diagram, it yielded a type B pyramid, or a pyramid with a gradual slope. Fire/burns (1:560:2720) also yielded this type of pyramid. However, the rate for ED visits from fire/burns was not high, showing that the scale of injury occurrence was also small.

In contrast, the ED visit rate for drowning was low, showing that it had a small scale of occurrence, but it was categorized as having a drastically high lethality, with an injury pyramid ratio of 1:1:5. When expressed in a pyramid diagram, it belonged to the type C pyramid, which is narrow and steep.

Injuries from MVCs (1:195:341), falls (1:127:329), and poisoning (1:114:214) showed medium levels of lethality, forming type A pyramids, which have an intermediate inclination compared to the previously mentioned 2 types of pyramids. The scale of occurrence of MVCs and falls was large, while the scale of poisoning occurrence was small.

Injuries from bicycle-related mechanisms had a relatively large scale, but their lethality was not high. While the scale of motorcycle-related injuries was not large, the lethality was relatively high.

\section{DISCUSSION}

The injury pyramids derived from the injury pyramid ratio calculated in this study showed varied sizes and shapes based on the intentionality and mechanism of injury. There are 3 major types of pyramids. Type C pyramids do not show significant differences between the rates of ED visitation, hospitalization, and death; in other words, injuries with high lethality result in a steep pyramid. Injuries with a low lethality, or those with much lower death rates compared to the ED visit rates, show a pyramid form with a gradual slope, known as a type B pyramid. Lastly, injuries showing a medium level of lethality form type A pyramids, with medium inclinations.

This categorization roughly corresponds to that developed by Wadman et al. [10], in which 3 major injury pyramids differ in shape based on lethality, with an inverted pyramid indicating high-lethality injuries, a rectangular pyramid indicating mid-level lethality injuries, and a typical pyramid indicating low-lethality injuries. Injuries due to the struck by/against 
mechanism had a low lethality rate, but the ED visit scale was large, thereby forming a type B pyramid in which the lower side of the pyramid was large and the top was very narrow. Injuries from drowning had a very high lethality rate but a small ED visit scale, resulting in a type $C$ pyramid with a steep and narrow shape. The results of Lee et al. [12] showed similar pyramid shapes for struck by/against injuries and drowning injuries. However, for fire/burn injuries, the ratio of the death rate to the ED visit rate was 1:2720 in this study, indicating a high incidence rate with low lethality, whereas Lee et al. [12] reported high lethality.

The mechanisms of struck by/against, MVCs, and falls had the highest number of ED visits, in accordance with the results of previous studies $[10,12]$. Similarly to previous studies, the MVCs and falls had the highest number of hospitalizations; however, the hospitalization rate from poisoning in this study was lower than in the results of previous studies. MVCs had the highest number of deaths, as in previous studies.

Comparisons of lethality according to different injury mechanisms provides insight on where to focus injury prevention initiatives. Injury mechanisms with high lethality can be prevented through primary prevention, which can lower the injury occurrence itself, or by making efforts to lower the lethality rate when injury occurs. In contrast, secondary or tertiary prevention efforts could be made through prompt and apt initial responses for injury mechanisms with medium lethality in order to reduce the length of hospitalization and the overall healthcare utilization for injuries.

Even if the scale of occurrence and lethality of an injury mechanism are low, attention should be paid to the severity of the sequelae of injuries. A study from Korea showed that injuries that occurred on the streets and were caused by falls or slips were more likely to result in disabilities [18]. Therefore, information on which injury mechanisms have higher healthcare utilization and higher medical costs can be an important factor for prioritizing resource allocation when making injury prevention and intervention policies. The comparison of intentional and unintentional injuries based on the pyramid types formulated in this study showed that intentional injuries had a steep type $C$ pyramid, indicating high lethality. The scale of occurrence, on the other hand, was larger for unintentional injuries, as in the results of international studies [12]. Injuries due to the struck by/against mechanism had low lethality but a large scale of occurrence, as well as a higher rate of hospitalization than the ED visitation rate, which illustrates that healthcare utilization and medical costs may be greater for such injuries. The lethality of injuries from MVCs and falls was medium, but the occurrence scale was large, indicating that they should be prioritized when making prevention and intervention policies. If only lethality is considered, the importance of large-scale mechanisms with greater medical service utilization may be overlooked.

Comparing the size and shape of injury pyramids can help yield insights into the scale and lethality of injuries; when this information is integrated with other data sources, more effective injury prevention and intervention policies can be made. For example, injuries that are not lethal can still cause severe physical or mental impairment, which can have negative longterm effects on a patient's health and quality of life. An index showing the long-term burden from injury is years lived with disability (YLD). According to the study on YLD from injuries conducted by Lee et al. [19], the lethality from injuries due to the struck by/against mechanism was low, but such injuries resulted in more YLD than injuries from bicycle or MVCs and poisoning, which had medium lethality.

This study used the data sources that best represented ED visits, hospitalizations, and deaths from injuries in Korea to create injury pyramids, but these sources had limitations that need to be taken into account. First, errors may have been made in the process of recording disease codes to define injuries. Furthermore, detailed information on injuries that were recorded as "etc." or "unknown" was not identified. Second, the data used in this study were not from the same year, and therefore are not representative values of the same year; the statistics on cause of death and EDIS were from 2015, while the data from the KNHDS was from 2011-2013.

Despite these limitations, this study succeeded in visualizing the intentions and mechanisms of injuries experienced by Korean adolescents as 3 types of pyramids, enabling a comparison of their scale and lethality at a glance. This can be used as an important foundation for establishing injury prevention policies. Future studies that include outpatient visit rates in the calculation of the injury pyramid ratio are expected to better estimate the burden to the medical system caused by injuries.

\section{ACKNOWLEDGEMENTS}

This work was supported by a research program funded by the Korea Centers for Disease Control and Prevention (fund code 2016-255). 


\section{CONFLICT OF INTEREST}

The authors have no conflicts of interest associated with the material presented in this paper.

\section{ORCID}

Hyejin Han http://orcid.org/0000-0002-1669-3177

Bomi Park https://orcid.org/0000-0001-5834-9975

Bohyun Park https://orcid.org/0000-0002-8538-9662

Namsoo Park https://orcid.org/0000-0002-8166-3943

Ju Ok Park https://orcid.org/0000-0002-1024-3626

Hyesook Park http://orcid.org/0000-0002-9359-6522

\section{REFERENCES}

1. Lim D, Lee WK, Park H. Disability-adjusted life years (DALYS) for mental and substance use disorders in the Korean Burden of Disease Study 2012. J Korean Med Sci 2016;31 Suppl 2: S191-S199.

2. Centers for Disease Control and Prevention. WISQARS ${ }^{\mathrm{TM}}$ - your source for U.S. injury statistics [cited 2018 Apr 5]. Available from: https://www.cdc.gov/injury/wisqars/facts.html.

3. Statistics Korea. Cause-of-death statistics; 2016 [cited 2018 Apr 5]. Available from: https://meta.narastat.kr/metasvc/index.do?confmNo=101054\&inputYear=2016 (Korean).

4. Freid VM, Makuc DM, Rooks RN. Ambulatory health care visits by children: principal diagnosis and place of visit. Vital Health Stat 13 1998;(137):1-23.

5. Yoon J, Oh IH, Seo H, Kim EJ, Gong YH, Ock M, et al. Disabilityadjusted life years for 313 diseases and injuries: the 2012 Korean Burden of Disease Study. J Korean Med Sci 2016;31 Suppl 2:S146-S157.

6. World Health Organization. Maternal, newborn, child and adolescent health: adolescent development [cited 2017 Dec 27]. Available from: http://www.who.int/maternal_child_adolescent/topics/adolescence/development/en/.

7. Global Burden of Disease Pediatrics Collaboration, Kyu HH, Pinho C, Wagner JA, Brown JC, Bertozzi-Villa A, et al. Global and national burden of diseases and injuries among children and adolescents between 1990 and 2013: findings from the Global Burden of Disease 2013 Study. JAMA Pediatr 2016; 170(3):267-287.

8. Statistics Korea. Statistics on the youth; 2016 [cited 2018 Apr 5]. Abailable from: http://kostat.go.kr/portal/eng/pressRe- leases $/ 1 /$ index.board? bmode $=$ read $\& a S e q=354961$.

9. Heinrich HW, Petersen D, Roos N, Brown JV, Hazlett S. Industrial accident prevention. 5th ed. New York: McGraw-Hill; 1980, p. 1-468.

10. Wadman MC, Muelleman RL, Coto JA, Kellermann AL. The pyramid of injury: using ecodes to accurately describe the burden of injury. Ann Emerg Med 2003;42(4):468-478.

11. Gallagher SS, Finison K, Guyer B, Goodenough S. The incidence of injuries among 87,000 Massachusetts children and adolescents: results of the 1980-81 Statewide Childhood Injury Prevention Program Surveillance System. Am J Public Health 1984; 74(12):1340-1347.

12. Lee LK, Fleegler EW, Forbes PW, Olson KL, Mooney DP. The modern paediatric injury pyramid: injuries in Massachusetts children and adolescents. Inj Prev 2010;16(2):123-126.

13. Korean Statistical Information Service. Annual report on the causes of death statistics 2015 [cited 2018 Apr 5]. Available from: http://kosis.kr/publication/publicationThema.do?pubcode $=$ YD (Korean).

14. Korea Centers for Disease Control and Prevention. Korean National Hospital Discharge In-depth Injury Survey, 2013 [cited 2018 Apr 5]. Available from: https://chs.cdc.go.kr/chs/injury/ sub06_cdc/sub06_01_cdc.do (Korean).

15. Korea Centers for Disease Control and Prevention. Guideline for Emergency Department-based Injury Sample In-depth Surveillance Data, 2013. Cheongju: Korea Centers for Disease Control and Prevention; 2013 (Korean).

16. Park HS, Park BH, Lee HA, Lim D, Park JO, Lee WK. Evaluation and integration of hospital-based injury surveillance systems in Korea; 2016 [cited 2018 Apr 5]. Available from: http://www. ndsl.kr/ndsl/search/detail/report/reportSearchResultDetail. do?cn = TRKO201600015684 (Korean).

17. Jung SY. Estimation and analysis of the incidence of suicidal attempts extrapolated from the emergency department based injury surveillance system [dissertation]. Seoul: Ewha Womans University; 2017 (Korean).

18. Park K, Eun SJ, Lee EJ, Lee CE, Park DY, Han K, et al. The incidence and patterns of unintentional injuries in daily life in Korea: a nationwide study. J Prev Med Public Health 2008;41(4): 265-271 (Korean).

19. Lee WK, Lim D, Park H. Disability-adjusted life years (DALYs) for injuries using death certificates and hospital discharge survey by the Korean Burden of Disease Study 2012. J Korean Med Sci 2016;31 Suppl 2:S200-S207. 